\title{
Theoretical Study of Tunable Optical Resonators in Periodic and Quasiperiodic One-Dimensional Photonic Structures Incorporating a Nematic Liquid Crystal
}

\author{
Youssef. Trabelsi ${ }^{1,2, *}$, Walid. Belhadj ${ }^{3}$, Naim. Ben Ali ${ }^{2,4}\left(\mathbb{C}\right.$ and Arafa H. Aly ${ }^{5}$ (i) \\ 1 Physics Department, College of Arts and Sciences in Muhail Asir, King Khalid University, \\ Abha 61421, Saudi Arabia \\ 2 Photovoltaic and Semiconductor Materials Laboratory, School of Tunis, University of Tunis El Manar, \\ National Engineering, Tunis 1002, Tunisia; na.benali@uoh.edu.sa \\ 3 Department of Physics, College of Applied Sciences, Umm Al-Qura University, Makkah 21421, Saudi Arabia; \\ wbbelhadj@uqu.edu.sa \\ 4 Department of Industrial Engineering, College of Engineering, University of Ha'il, \\ Ha'il City 2440, Saudi Arabia \\ 5 TH-PPM Group, Physics Department, Faculty of Sciences, Beni-Suef University, Beni Suef 62521, Egypt; \\ arafaaly@aucegypt.edu \\ * Correspondence: ytrabelsi@kku.edu.sa
}

check for updates

Citation: Trabelsi, Y.; Belhadj, W.; Ben Ali, N.; Aly, A.H. Theoretical Study of Tunable Optical Resonators in Periodic and Quasiperiodic One-Dimensional Photonic Structures Incorporating a Nematic Liquid Crystal. Photonics 2021, 8, 150. https://doi.org/10.3390/ photonics 8050150

Received: 13 March 2021

Accepted: 7 April 2021

Published: 1 May 2021

Publisher's Note: MDPI stays neutral with regard to jurisdictional claims in published maps and institutional affiliations.

Copyright: (c) 2021 by the authors. Licensee MDPI, Basel, Switzerland. This article is an open access article distributed under the terms and conditions of the Creative Commons Attribution (CC BY) license (https:// creativecommons.org/licenses/by/ $4.0 /)$.

\begin{abstract}
In this work, the transfer matrix method (TMM) is employed to investigate the optical properties of one-dimensional periodic and quasiperiodic photonic crystals containing nematic liquid crystal (NLC) layers. This structure is expressed as (ABC)J(CBA)J and made of alternated layers of isotropic dielectrics $\mathrm{SiO}_{2}$ (A), $\mathrm{BGO}$ (B) and nematic liquid crystal (C). The simulation study shows that the proposed ternary configuration exhibits tunable defect mode within the photonic band gap (PBG) that can be manipulated by adjusting the thicknesses of NLC layers in order of the periodic lattice. In addition, the optimized structure permits for strong confinement light giving rise to an optical microcavity. The application of an applied voltage into NLC layers enables improving the sensitivity by guiding the local defect mode. It has been also shown that by applying quasiperiodic inflation according to Rudin Shapiro Sequence (RSS) scheme to main periodic structure, several tunable resonant modes appear within the PBG. The presence of such sharp resonant peaks reflects that the quasiperiodic NLC-based structure behaves like multiple microcavites with strong lightmatter coupling.
\end{abstract}

Keywords: transfer matrix method (TMM); photonic crystal (PC); quasicrystals; nematic liquid crystal (NLC); rudin shapiro sequence (RSS)

\section{Introduction}

Photonic crystals (PCs) discovered by Yablonovitch in 1987 [1] are artificial composites of insulating or metallic materials. In PCs the electromagnetic waves are prohibited to propagate in certain frequency ranges called photonic band gap (PBG) and are allowed modes in other ones. These features leading to control light within certain wavelength regions are very useful for the implementation of optoelectronic devices [2-5]. Further, [6] has explained the whole theory that represents the background about confined modes in photonic band gap of multilayer nanostructures. Photonic quasicrystals (PQCs) are a new field of photonic band gap materials. It is an intermediate medium between the periodic and disordered crystals that possess a perfect long-range orientational order without translation symmetry [7-10]. The quasiperiodic 1D-PCs are the simplest form of PQCs, and they are built according to deterministic inflation rules of quasiperiodic sequence such as Fibonacci, Thue Morse, Cantor, Rudin Shapiro [11-13]. 
Recently, intensive studies used quasicrystals and photonic amorphous materials instead of ordinary PC in order to improve the performance of an optical devise [14-16]. It opens structural microcavities within the main PBG, which improves the photonic band gap properties [17]. The rupture of periodicity offers one defect within the PCs called cavity that is able to confine light in which the field distribution exhibits a strong localization and opens new narrow peaks of high transmissivity within the PBG [18-24].

The incorporation of external materials into pure periodic PCs such as superconductor [25-27], metal [28], magnetized plasma [29], semiconductor [30] and liquid crystal [31-33] permits introducing a localized mode that is very useful to develop compact optical resonators. Such a photonic resonator is highly sensitive to external conditions such as temperature [34], pressures [35] and self-organization of multilayered stacks [36].

Among the above-mentioned materials, the insertion of liquid crystal into PCs has attracted much attention due to promising applications in switching and filtering operations such as optical switches and antireflection coating [37-40]. Further, [41] has proposed an optical beam steering device, operating at a wavelength of $1550 \mathrm{~nm}$ to drive the light by the new fascinating LC and metastructures/metamaterials. Also, Chong Wu et al. [42] have studied optical properties of one-dimensional PC incorporting nematic liquid crystal (NLC). They demonstrated tunability of transmission spectrum by modulation of the nematic director and applied voltage of NLC slab. Commonly, the liquid crystal (LC) is a substance between the crystal solid and the isotropic liquid that possesses significant characteristics of both crystalline solid and liquid. This intermediate state exhibits some physical properties not found in both liquid and solid states. They have regular properties well-ordered by magnetic or electric fields. They possess moving molecules with limited rotational mobility. There are many types of liquid crystals, which differ according to the arrangement of their molecules: Semitic, Nematic and Cholesteric. Further, the planar Nematic LC is extensively used as a central defect into one-dimensional (1D) PCs due to its ability to obtain a tunable Photonic bang gap (PBG). This type of hybrid LC-Photonic crystals exhibits an excellent ability to control the propagation of light modes. The paper discusses the optical properties of $1 \mathrm{D}$ periodic and quasiperiodic photonic crystals containing Nematic liquid crystals. The studied structure in this work is expressed as $(\mathrm{ABC})^{\mathrm{J}}(\mathrm{CBA})^{\mathrm{J}}$ and made of alternated layers of isotropic dielectrics $\mathrm{SiO}_{2}$ (A), $\mathrm{BGO}$ (B) and nematic liquid crystal (C) materials. The simulation study is based on the transfer matrix method. In addition, the paper discusses the possibility of improving the photonic bandgap and resonant peaks within the band gap in 1D photonic crystals and quasicrystals using the transfer matrix approach. A numerical optimization of transmitted resonance peak is achieved by applying an external voltage to the nematic liquid layers and adjusting the properties of incorporated nematic liquid layer, showing a significant improvement of photonic bandgap and resonant peaks. To improve the confinement of light by open multiple microcavities, multilayered stacks with constituents arranged according to Rudin Shapiro Sequence (RSS) scheme is followed.

\section{Theoretical Model}

The one-dimensional periodic and quasiperiodic multilayered stacks are built according to the periodic modulation of the dielectric constants and the substitution rule of quasiperiodic sequence, respectively. It is well known that these kind of quasicrystals with perfect long-range order and without translational symmetry exhibit self-similarity properties. The transfer matrix method (TMM) has been employed to investigate the optical response of these stratified media. The TMM approach takes into consideration several important potential physical phenomena. It constitutes an efficient tool to compute power distribution as well as transmission, reflection and absorption coefficients in stratified structures. Within the TMM approach, the amplitudes of the input and output electric fields are expressed by the matrix formula as $[43,44]$.

$$
\left[\begin{array}{l}
E_{0}^{+} \\
E_{0}^{-}
\end{array}\right]=\prod_{i=1}^{m} \frac{C_{j}}{t_{j}}\left[\begin{array}{l}
E_{m+1}^{+} \\
E_{m+1}^{-}
\end{array}\right]
$$


where $C_{j}$ denotes the transfer matrix of the jth layer, and it is given for both TM and TE polarizations by $[43,44]$ :

$$
C_{j}=\left(\begin{array}{cc}
\exp \left(i \varphi_{j-1}\right) & r_{j} \exp \left(-i \varphi_{j-1}\right) \\
r_{j} \exp \left(i \varphi_{j-1}\right) & \exp \left(-i \varphi_{j-1}\right)
\end{array}\right)
$$

Here, $\varphi_{j-1}$ which denotes the phase in $(j-1)$ th layer can be expressed from $[43,44]$ as follows:

$$
\varphi_{j-1}=\frac{2 \pi}{\lambda} \hat{n}_{j-1} d_{j-1} \cos \theta_{j-1}
$$

where $\mathrm{d}_{j-1}, \hat{\mathrm{n}}_{\mathrm{j}-1}$ and $\theta_{\mathrm{j}-1}$ are respectively the thickness of the refractive index and incident angle of the $(j-1)$ th layer. The Fresnel coefficients $t_{j}$ are $r_{j}$ can be written as follows $[43,44]$ :

For TE or p-polarization:

$$
\begin{aligned}
& r_{j p}=\frac{n_{j-1} \cos \theta_{j}-n_{j} \cos \theta_{j-1}}{n_{j-1} \cos \theta_{j}+n_{j} \cos \theta_{j-1}} \\
& t_{j s}=\frac{2 n_{j-1} \cos \theta_{j-1}}{n_{j-1} \cos \theta_{j}+n_{j} \cos \theta_{j-1}}
\end{aligned}
$$

For TM or s-polarization:

$$
\begin{array}{r}
r_{j s}=\frac{n_{j-1} \cos \theta_{j-1}-n_{j} \cos \theta_{j}}{n_{j-1} \cos \theta_{j-1}+n_{j} \cos \theta_{j}} \\
t_{j s}=\frac{2 n_{j-1} \cos \theta_{j-1}}{n_{j-1} \cos \theta_{j-1}+n_{j} \cos \theta_{j}}
\end{array}
$$

For both modes, the transmittance is given by $[40,41]$ :

$$
\begin{aligned}
& \mathrm{T}_{\mathrm{s}}=\operatorname{Re}\left(\frac{\hat{\mathrm{n}}_{\mathrm{m}+1} \cos \theta_{\mathrm{m}+1}}{\hat{\mathrm{n}}_{0} \cos \theta_{0}}\right)\left|\mathrm{t}_{\mathrm{S}}\right|^{2} \\
& \mathrm{~T}_{\mathrm{p}}=\operatorname{Re}\left(\frac{\hat{\mathrm{n}}_{\mathrm{m}+1} \cos \theta_{\mathrm{m}+1}}{\hat{\mathrm{n}}_{0} \cos \theta_{0}}\right)\left|\mathrm{t}_{\mathrm{P}}\right|^{2}
\end{aligned}
$$

The refractive index of an NLC layer is given by [45,46]:

$$
n_{C L}=\left(\begin{array}{ccc}
n_{o}^{2} \sin ^{2} \psi+n_{e}^{2} \cos ^{2} \psi & \left(n_{e}^{2}-n_{o}^{2}\right) \cos \psi \sin \psi & 0 \\
\left(n_{e}^{2}-n_{o}^{2}\right) \cos \psi \sin \psi & n_{o}^{2} \cos ^{2} \psi+n_{e}^{2} \sin ^{2} \psi & 0 \\
0 & 0 & n_{o}^{2}
\end{array}\right)
$$

where $n_{o}$ and $n_{e}$ are the ordinary and extraordinary refractive index of NLC, respectively. Here, $\psi$ denotes the the tilt angle of NLC molecules.

For ordinary wave, the refractive indices are isotropic and can be expressed as [47]:

$$
n_{C L}=\frac{n_{o} n_{e}}{\sqrt{n_{o}^{2} \sin ^{2} \psi+n_{e}^{2} \cos ^{2} \psi}}
$$

When an external voltage is applied to an NLC slab, the nematic liquid crystal molecules can be simply reoriented. From the Ossen Frank elastic theory the direction of NLC slabs exhibits inhomogeneous distribution under applied electric fields.

The relations between the applied voltage $\mathrm{V}$ and tilt angle of NLC molecules can be written as follows [48]:

$$
\frac{V}{V_{c}}=\frac{2}{\pi} \int_{0}^{\frac{\pi}{2}} \sqrt{\frac{1+k \eta^{2} \sin ^{2} \psi}{1-\eta^{2} \sin ^{2} \psi}} d \psi
$$


Here, $\eta=\sin \alpha_{\mathrm{m}}$ with $\alpha_{\mathrm{m}}$ is the maximum tilt angle in the NLC slab. Note that $k=\frac{k_{3}-k_{1}}{k_{1}}$ with $k_{1}$ and $k_{3}$ are the Frank elastic constants and $V_{c}$ denotes the threshold voltage. The plane geometry of the cell construction consisting of periodically modulated of three different layers is shown in Figure 1. The heterolyers follows the distribution: $\mathrm{H}(\mathrm{LH})^{J}[\mathrm{LCN}] \mathrm{H}(\mathrm{LH})^{J}$. Here, J determines the number of period of stacks. Let us note that the stratified multilayer stacks are embedded in air background with refractive index $n_{\text {air }}=1$.
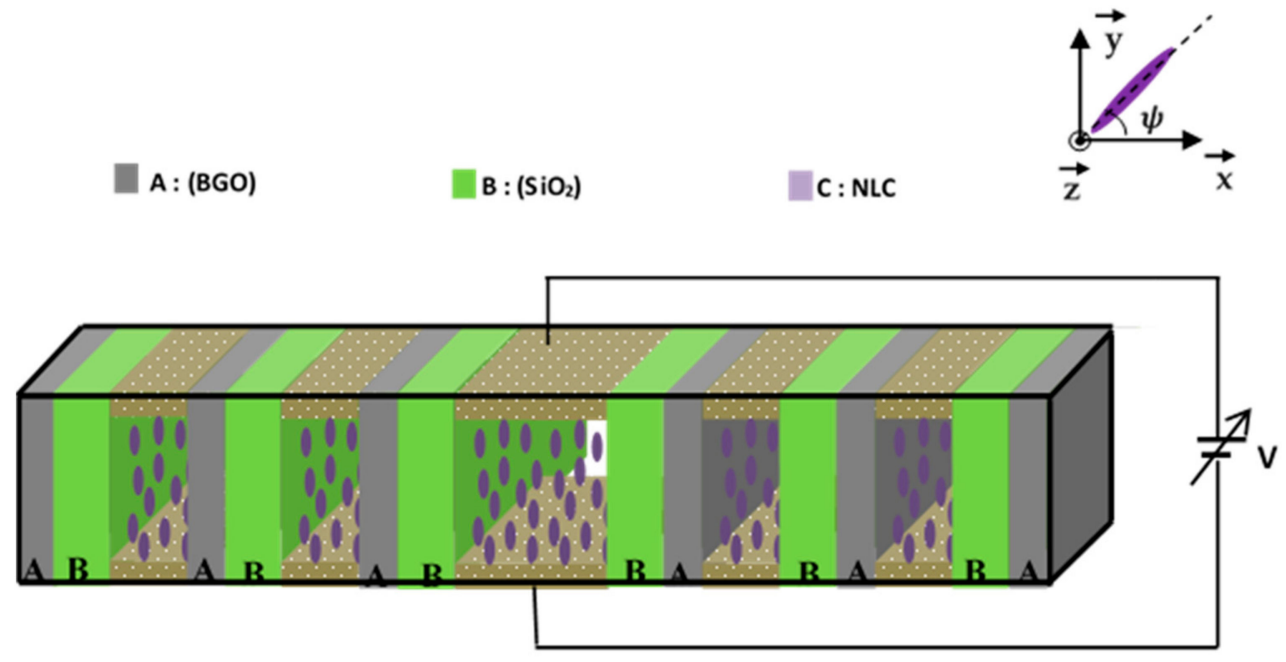

Figure 1. Stratified multilyered stack of three layers containing nematic liquid crystal (NLC) slabs designed as: $(\mathrm{ABC})^{3}(\mathrm{CBA})^{3}$ with $\mathrm{SiO}_{2}(\mathrm{~A}), \mathrm{BGO}(\mathrm{B})$ and $\mathrm{NLC}(\mathrm{C})$ connected to external voltage.

\section{Result and Discussion}

We will employ the TMM approach to investigate the spectral behaviors of 1D NLCbased photonic structures versus parameters related to NLC constituent layers such as thickness, tilt angle and applied voltage.

First, the optical response of a regular 1D NLC-PC with the configuration (ABC) $)^{5}$ $(\mathrm{CBA})^{5}$ is analyzed. In this numerical calculation, layers $\mathrm{A}$ and $\mathrm{B}$ are are chosen to be silica $\left(\mathrm{SiO}_{2}\right)$ and $\mathrm{Bi}_{4} \mathrm{Ge}_{3} \mathrm{O}_{12}$ (BGO) materials, respectively. The corresponding refractive indices and thicknesses of layers $A$ and $B$ are $\left(n_{A}=2.3, d_{A}=287.5 \mathrm{~nm}\right)$ and $\left(n_{B}=1.45, d_{B}=181\right.$. $25 \mathrm{~nm}$ ). The layer $C$ corresponds to the nematic liquid crystal (NLC) with ordinary and extraordinary refractive indices $n_{0}=1.500$ and $n_{e}=1.689$, respectively [44]. The thickness of the NLC layer and the threshold applied voltage are taken to be $d_{C}=165 \mathrm{~nm}$ and $V_{C}=0.96$ volts, respectively. For the nematic liquid crystal, we choose the following elastic constants: $\mathrm{K}_{1}=12 \mathrm{pN}, \mathrm{K}_{2}=9 \mathrm{pN}$ and twist, $\mathrm{K}_{3}=19.5 \mathrm{pN}$ as in [48].

The design of $1 \mathrm{D} \mathrm{PhC}$ consisting of multilayer thin films of either isotropic or anisotropic materials has widely studied. The design of 1D PC containing NLC with desired property needs the employment of spacers around the NLC lattice. It is worth noting that spacers can be practically used to sandwich the NLC layer with the stacked dielectric multilayers. Further, the spacers will not affect the transmission characteristics through the studied 1D NLC PhC, and hence the spacers will not be taken into consideration in the simulation process.

Figure 2 shows the linear 3D transmission spectrum versus wavelength and incident angle (rad) for different rotational angles of NLC. It is clearly seen that the considered structure exhibits a defect mode within the PBG region $460-550 \mathrm{~nm}$ for both rotational angles of the NLC. This defect mode shifts towards higher wavelengths as the incidence angle increases. This behavior is due to the increase of the refractive index, the anisotropy and mode coupling in the structure and the applied voltages on NLC layers in order to keep the same thicknesses of all heterolayers. The configuration of the proposed $1 \mathrm{D} \mathrm{PhC}$ 
containing nematic liquid crystal acts as an optical microcavity; this is due to the appearing of strong optical confinement in the corresponding transmittance spectra.

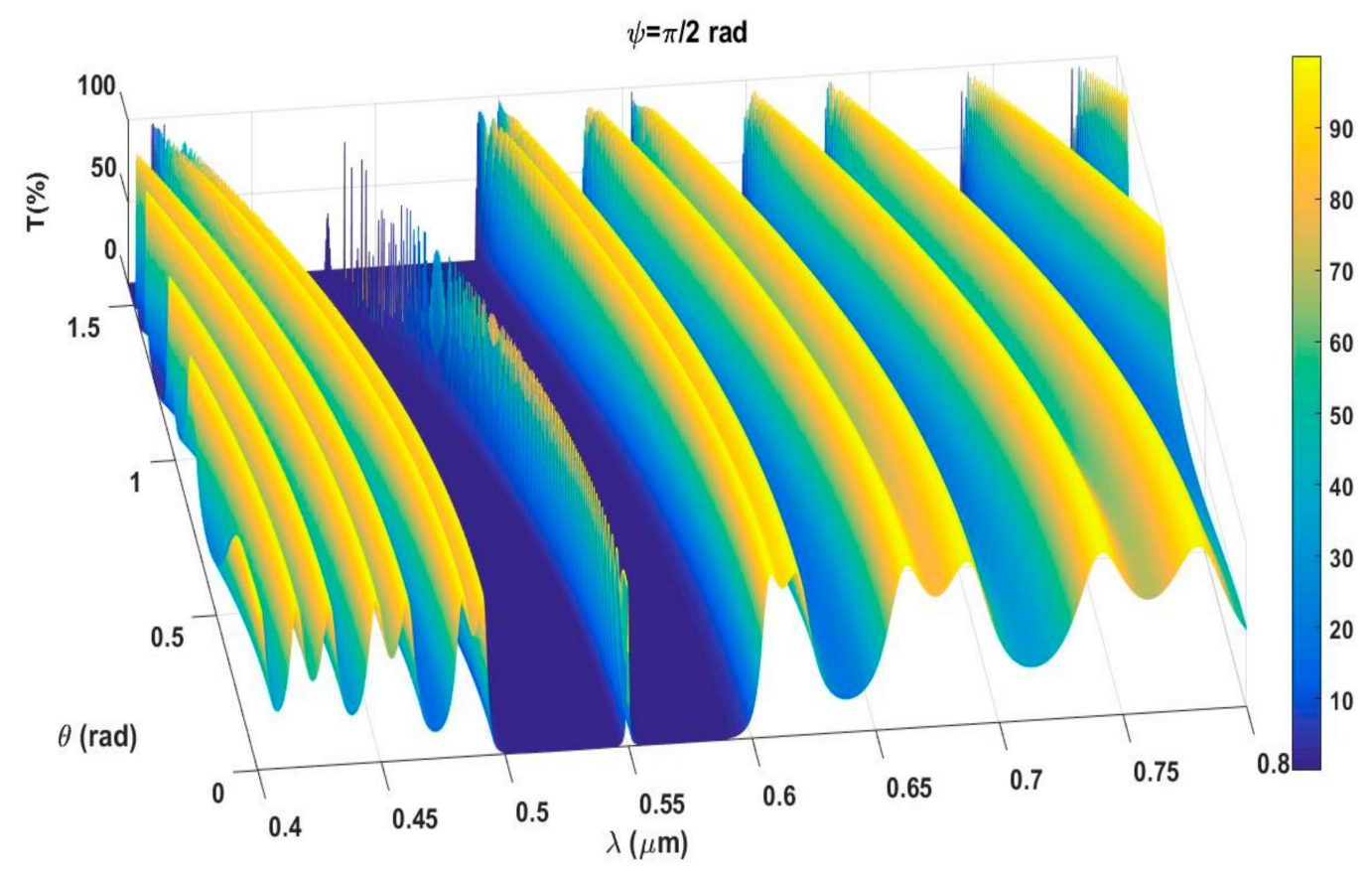

Figure 2. Linear transmission spectrum of $(\mathrm{ABC})^{5}(\mathrm{CBA})^{5}$ configuration at different rotational angles of NLC.

Figure 3 shows that the 2D allowed structural modes at two distinct tilt angles of the NLC layers $\psi=0\left(V_{C}=0\right)$ and $\psi=\frac{\pi}{2}\left(V_{C}=V_{C \max }\right)$. It can be clearly appreciated from Figure 3 that there are sharp narrow peaks within the main photonic band gap (PBG). When $\psi$ increases from 0 to $\pi / 2$, the spectral position of this mode moves toward longer wavelengths. This behavior is due to the decoupling between the main constituents NLC/PC within the present stacks.

(a) $\psi=0$

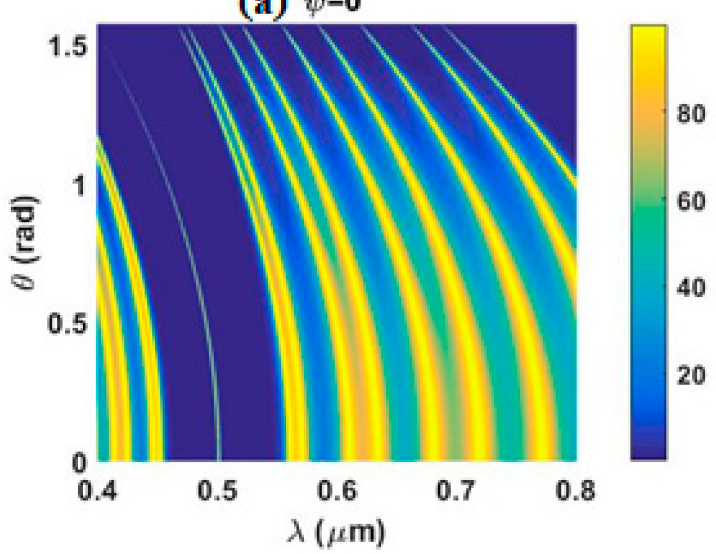

(b) $\psi=\pi / 2 \mathrm{rad}$

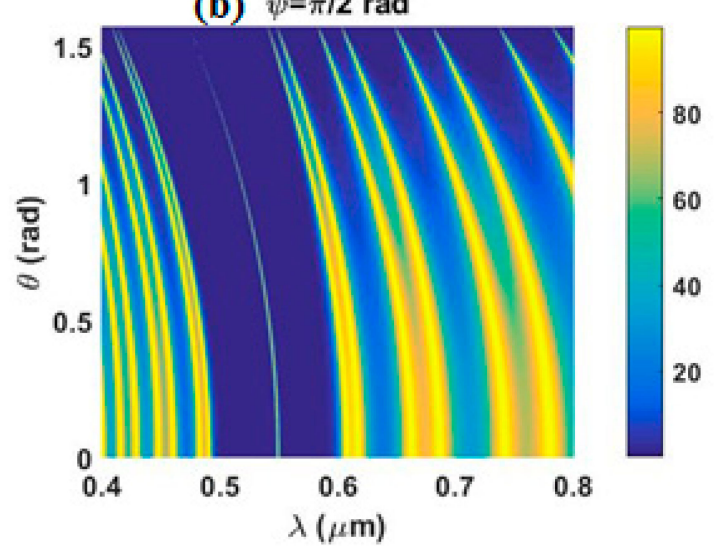

Figure 3. Electric field profiles spectra through 1D-hybrid NLC/photonic crystal (PC) stacks as function of wavelength and wave incidence angle and for: (a) $\psi=0$ and (b) $\psi=\frac{\pi}{2}$.

To further explore the properties of defect modes of our regular NLC-based photonic structure: $[\mathrm{ABC}]^{\mathrm{J}}[\mathrm{BAC}]^{\mathrm{J}}$ in visible spectral range, we calculate the transmission spectra at normal incidence for different number of periods $\mathrm{J}=5$ and $\mathrm{J}=8$. Figure 4 shows that the amplitude of resonant peak can be modulated by varying the number of period $\mathrm{J}$ of the photonic crystal. We achieved a resonant peak with amplitude of $100 \%$ for $\mathrm{J}=5$ and $75 \%$ for 
$\mathrm{J}=8$. So the present NLC-based photonic structure acts as an optical microcavity in which light is strongly localized. Thus, the differences between the spectra of Figure $4 \mathrm{a}, \mathrm{b}$ can be attributed to the coincidence of wave propagation at reference wavelength and dielectric contrast effects. Thus, an efficient heterostructure with a sharply defined microcavity for the least number of layers has been acheived.
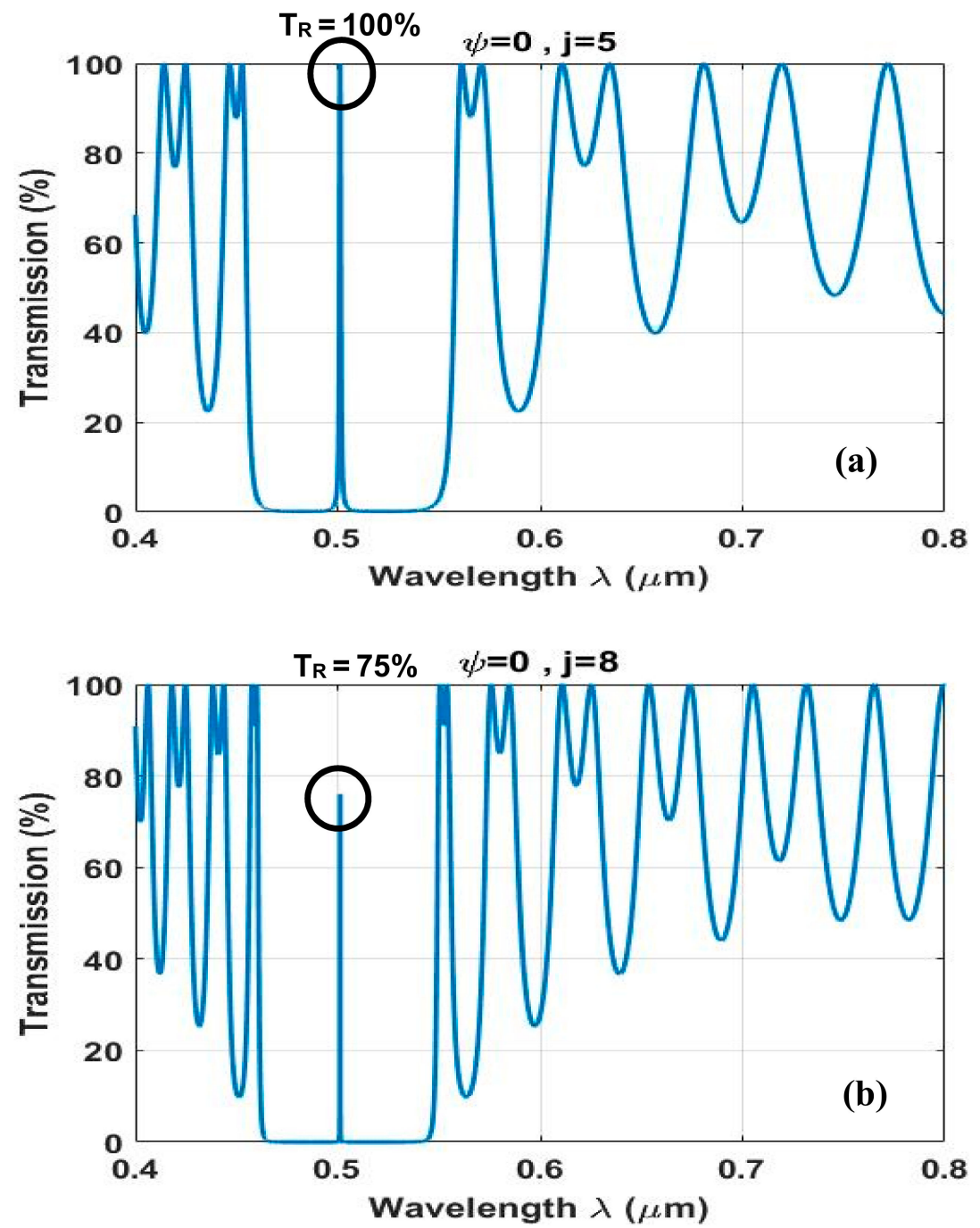

Figure 4. Transmission spectra of a quarter wave hybrid NLC/PC: $[A B C]^{J}[B A C]^{J}$ dielectric heterostructure as function of wavelength with $\mathrm{J}$ is set to be $5(\mathbf{a})$ and $8(\mathbf{b})$, respectively.

The reason the amplitude of resonant peak can be modulated by varying the number of period of the photonic crystal can be understood by the constructive and destructive interference of multiple reflections arising from the wave fronts generated at each interfaces separating two different materials with refractive index $n_{1}$ and $n_{2}$. Generally, by increasing the number of layers of the structures, more and more transmission zones diminish gradually and some of them approach zero transmission. For larger periods, the multiple reflectance increases and the amplitude of wave propagation through the material is slightly attenuated. In Figure 4, the remaining peaks appear around the main band gap and represent the defect modes with nonzero transmission. Such a defect mode is a bound state out of continuum and is created due to the resonances.

Figure 5 exhibits the transmittance spectra at normal incidence for various thicknesses $d_{\mathrm{NLC}}$ of NLC layers. The figure shows that the spectral position of the resonant peak is strongly affected by the variation of $d_{\mathrm{NLC}}$. The peak height is not influenced by the variation 
of $d_{\mathrm{NLC}}$, while its spectral position is shifted toward higher wavelengths by increasing $d_{\mathrm{NLC}}$. Hence, the proposed NLC-based PC configuration offers a tunable bihavior against the thickness of the NLC layers. Such structures are suitable tools for designing of tunable narrowband filters and high sensitive sensor devices. Further, the output spectrum of the proposed optical resonators exhibit a quality factor that gradually decreases as $d_{\text {NLC }}$ increases. Therefore the quality factor values of the obtained resonators are about 842.10 , $827.72,812.18$ and 798 for $\mathrm{d}_{\mathrm{NLC}}$ set to be $150 \mathrm{~nm}, 155 \mathrm{~nm}, 160 \mathrm{~nm}$ and $165 \mathrm{~nm}$, respectively.

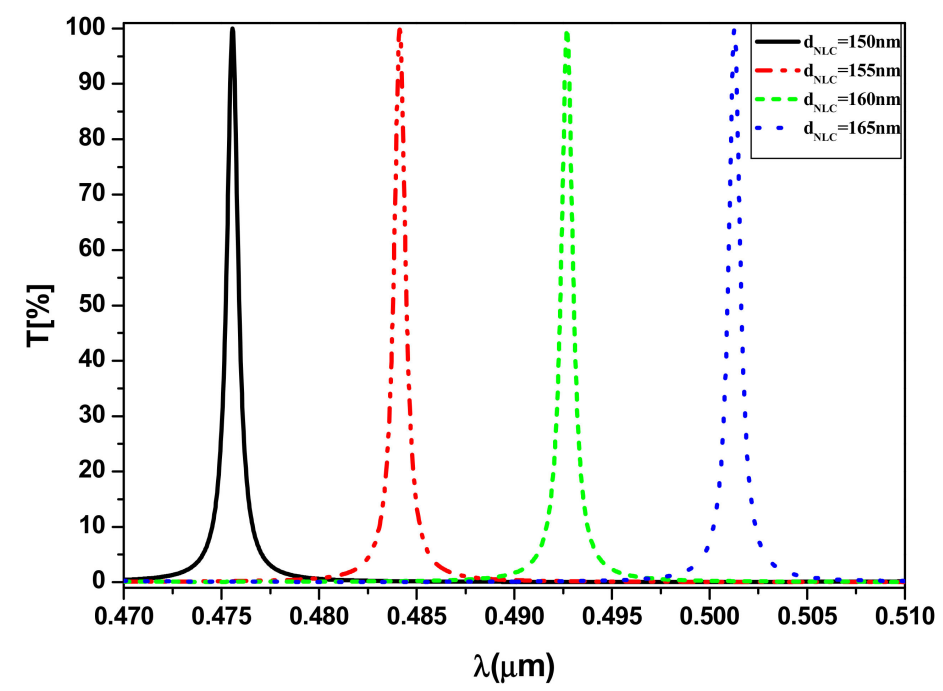

Figure 5. Evolution of resonant peak of regular symmetrical NLC-PC sample as the function of wavelengths for different thickness $\mathrm{d}_{\mathrm{NLC}}$ of NLC.

Next, we analyzed the influences of applied external voltage on the properties of the defect mode of our NLC-based photonic structure. Figure 6 displays the dependence of resonant peak with applied voltages bias. Note that the spectral position of the peak shifts towards longer wavelengths when the applied voltage increases. This behavior is due to the fact that the reorientation of the director is sensitive to refractive index of NLC. Moreover, the orientation of the molecules of NLC is controlled under the variation of applied electric field. So, the increase of applied voltage leads to the gradual increase of the energy of the electric field. In addition, the function of electric field play a leading role when the applied voltage exeeds the threshold value.

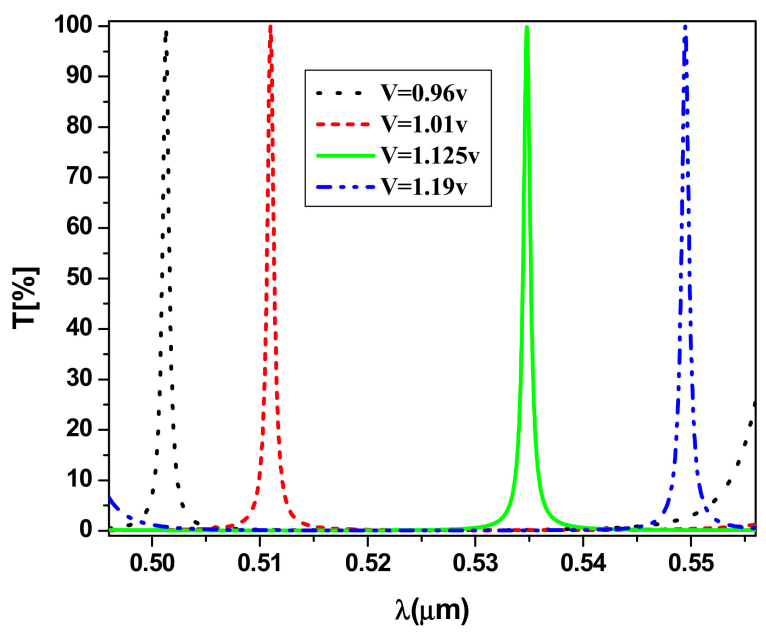

Figure 6. Evolution of resonant peak of regular symmetrical NLC/PC sample as the function of wavelengths for different external applied voltages V. Here, $\mathrm{d}_{\mathrm{NLC}}$ are set to be $0.96 \mathrm{v}, 1.01 \mathrm{v}$, $\mathrm{V}=1.125 \mathrm{v}$ and $\mathrm{V}=1.19 \mathrm{v}$, respectively. 
In order to improve the characteristics of designed microstructure, we introduce a simple aspect of disorder in the arrangement of our initial structure that leads to a new photonic quasicrystal (PQC). This new NLC-based PQC is built according to Rudin Shapiro Sequence (RSS) scheme that leads to the development of a regular polychromatic filter with improved performances. Figure 7 shows the transmittance spectra at normal incidence of the NLC. It is obvious from this figure that the stucture exhibits two resonant modes with maximum amplitude $(100 \%)$ called microcavity peaks (m-peaks) at $\lambda_{0}=0.5 \mu \mathrm{m}$ and $\lambda_{1}=0.72 \mu \mathrm{m}$, repectively. The spectrum exhibits secondary peaks with lower amplitudes (about $85 \%$ ) that appear at harmonic wavelengths $\lambda=0.41 \mu \mathrm{m}$ and $\lambda=0.65 \mathrm{~mm}$, respectively. In addition, we note the presence of PBGs in which the propagation wave is forbidden. The RSS configuration exhibits sharp and multiple resonnat peaks compared to the symmetrically chirped-NLC based PC configuration.

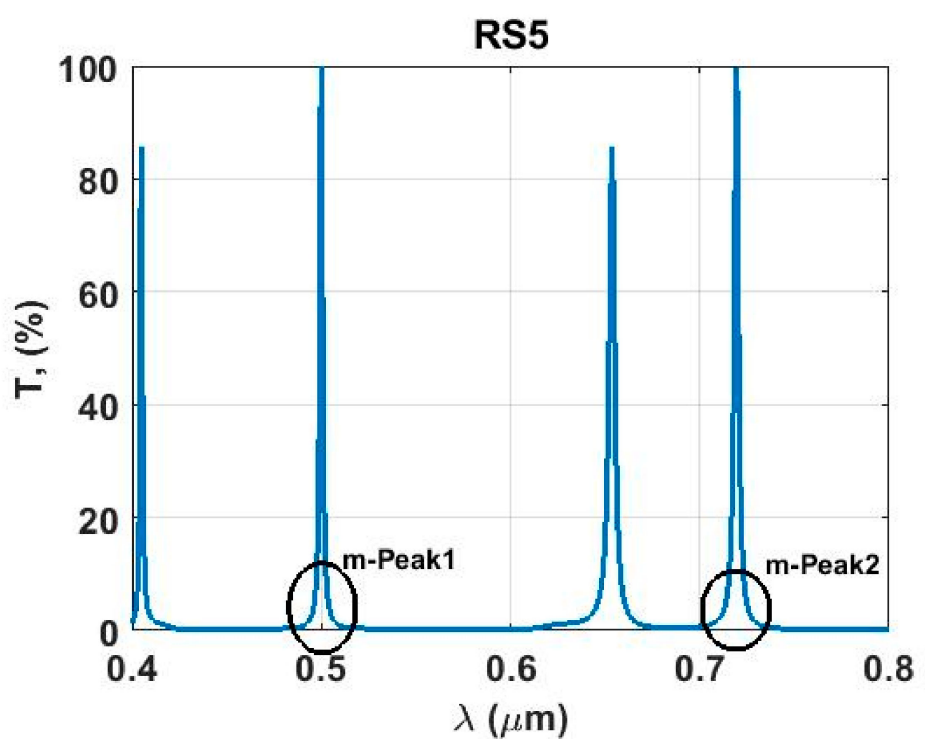

Figure 7. Transmission spectrum of a quarter wave hybrid NLC/PC materials built according the fifth order of RSS as function of wavelength.

Let us consider hybrid NLC/PC structures built according the fifth order of RSS with applied external voltage. We studied the effect of change in applied voltage on RSS transmittance spectra. In Figure 8, we have plotted the wavelength-dependent transmittance at different voltages $\mathrm{V}=0.96 \mathrm{v}, 1.01 \mathrm{v}, 1.125 \mathrm{v}$ and $1.19 \mathrm{v}$, respectively. By increasing $\mathrm{V}$, the obtained resonances shifted to a longer wavelength region. It is obvious that from all spectrums the amplitude of given resonance peaks increases with set voltages. This increase in peak height means a complete resonance at the defect mode with perfect organization orientational of nematic liquid crystal molecules. Then, such set voltages are very important to calibrate the hybrid NCL/PC structure by adjusting the characteristics of resonance.

Now we discuss the influence of set voltages on transmittance spectra at reference wavelength $\lambda_{0}=0.5 \mu \mathrm{m}$. Figure 9 shows the dependence of the given resonance peak on the wavelength in the region near the reference wavelength. Looking at the spectrum, all amplitudes are approximately equal for the different set voltages. Referring to this Figure, an obvious shift is well-observed when the set voltage of the NLC layer increases from $\mathrm{V}=0.96 \mathrm{v}$ to $\mathrm{V}=1.19 \mathrm{v}$. The former cited shift is caused by the increase of the resonant cavities, which permits the localization of higher wavelength. This property improves the capability of the proposed NLC photonic crystals for optical and sensing applications. 


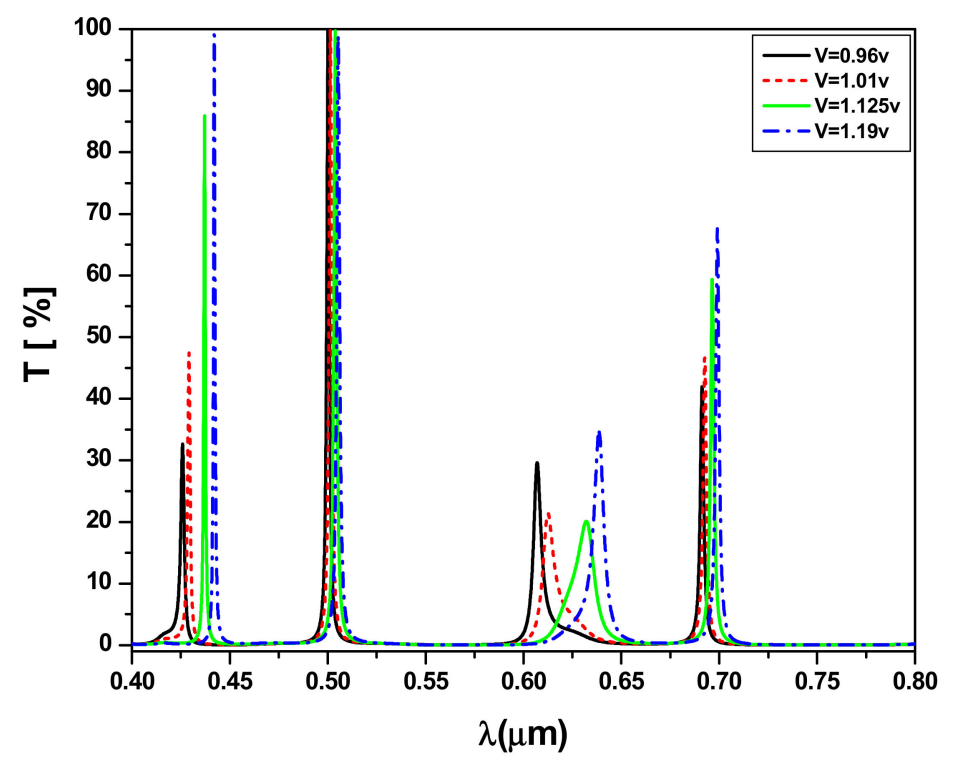

Figure 8. Dependence of the transmittance on the wavelength of hybrid PC with incorporating NLC built according the fifth order of RSS for different values of applied external voltage.

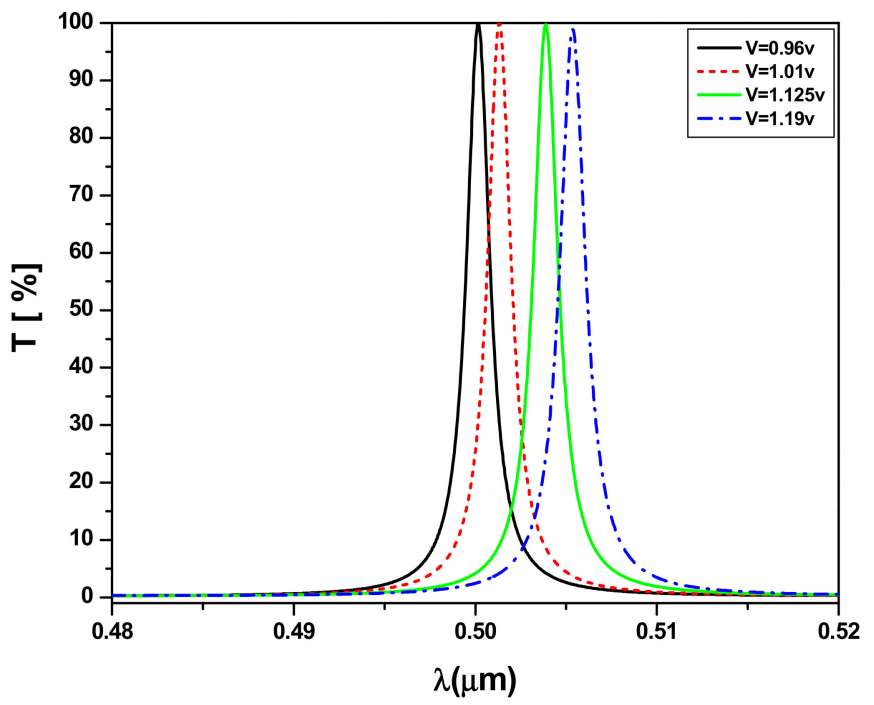

Figure 9. Evolution of the transmittivity spectra with wavelength of a hybrid PC with incorporating NLC built according the fifth order of RSS for different values of applied external voltage.

\section{Conclusions}

To conclude, TMM method was employed to investigate the transmission properties of 1D NLC-based PC resonant structures with periodic and quasiperiodic patterns. The main stacks consist of alternate photonic materials and the NLC. It was shown that the NLC-based PC structure with periodic configuration exhibits a sharp cavity mode within the PBG. Our simulations revealed that both the amplitude and the resonance frequency of such cavity modes are strongly dependent on the optogeometrical parameters of the NLC layers. Such structures are suitable tools for designing tunable narrowband filters and high-sensitive sensor devices. In addition, the rearrangement of the NLC-based PC structure according to Rudin Shapiro Sequence (RSS) scheme leads to the opening of multiple induced defect modes within the PBG, and the structure behaves like an optical resonator with coupled multiple microcavities. 
Author Contributions: Conceptualization, Y.T.; Formal analysis, Y.T.; Funding acquisition, W.B.; Investigation, N.B.A.; Methodology, N.B.A.; Software, A.H.A.; Supervision, A.H.A. All authors have read and agreed to the published version of the manuscript.

Funding: This research received no external funding.

Acknowledgments: The authors are thankful to the Deanship of Scientific Research-Research Center at King Khalid University in Saudi Arabia for funding this research (code number: R.G.P.1/182/41).

Conflicts of Interest: The authors declare no conflict of interest.

\section{References}

1. Yablonovitch, E. Inhibited Spontaneous Emission in Solid-State Physics and Electronics. Phys. Rev. Lett. 1987, 58, 2059-2062. [CrossRef] [PubMed]

2. Yablonovitch, E. Photonic band-gap structures. Josa B 1993, 10, 283-295. [CrossRef]

3. Talebzadeh, R.; Soroosh, M.; Kavian, Y.S.; Mehdizadeh, F. Eight-channel all-optical demultiplexer based on photonic crystal resonant cavities. Optik 2017, 140, 331-337. [CrossRef]

4. Bhattacharya, P.K. Photonic Crystal Devices. J. Phy. D Appl. Phy. 2007, 40, E01.

5. Trabelsi, Y. Tunable properties of omnidirectional band gap based on photonic quasicrystals containing superconducting material. Opt. Quantum Electron. 2021, 53, 1-10. [CrossRef]

6. Lio, G.E.; Palermo, G.; Caputo, R.; De Luca, A. A comprehensive optical analysis of nanoscale structures: From thin films to asymmetric nanocavities. Rsc Adv. 2019, 9, 21429-21437. [CrossRef]

7. Steurer, W.; Widmer, D.S. Photonic and phononic quasicrystals. J. Phys. D Appl. Phys. 2007, 40, R229. [CrossRef]

8. Edagawa, K. Photonic crystals, amorphous materials, and quasicrystals. Sci. Technol. Adv. Mater. 2014, 15, 034805. [CrossRef]

9. Levine, D.; Steinhardt, P.J. Quasicrystals: De_nition and structure. Phys. Rev. B 1986, 34, 596-616. [CrossRef]

10. Vardeny, Z.V.; Nahata, A.; Agrawal, A. Optics of photonic quasicrystals. Nat. Photonics 2013, 7, 177-187. [CrossRef]

11. Sahel, S.; Amri, R.; Bouaziz, L.; Gamra, D.; Lejeune, M.; Benlahsen, M.; Bouchriha, H. Optical filters using Cantor quasi-periodic one dimensional photonic crystal based on $\mathrm{Si} / \mathrm{SiO}_{2}$. Superlattices Microstruct. 2016, 97, 429-438. [CrossRef]

12. Trabelsi, Y.; Benali, N.; Bouazzi, Y.; Kanzari, M. Microwave transmission through one-dimensional hybrid quasi-regular (fibonacci and Thue-Morse)/periodic structures. Photonic Sens. 2013, 3, 246-255. [CrossRef]

13. Trabelsi, Y.; Bouazzi, Y.; Benali, N.; Kanzari, M. Narrow stop band optical filter using one-dimensional regular Fibonacci/Rudin Shapiro photonic quasicrystals. Opt. Quantum Electron. 2016, 48, 1-9. [CrossRef]

14. Segovia-Chaves, F.; Vinck-Posada, H.; Trabelsi, Y.; Ben Ali, N. Transmittance spectrum in a one-dimensional photonic crystal with Fibonacci sequence superconductor-semiconductor. Opt. Int. J. Light Electron Opt. 2020, 217, 164803. [CrossRef]

15. Trabelsi, Y. Output multichannel optical filter based on hybrid photonic quasicrystals containing a high-Tc superconductor. Photonics Nanostruct. Fundam. Appl. 2019, 36, 100724. [CrossRef]

16. Ben Ali, N.; Dhasarathan, V.; AlSaif, H.; Trabelsi, Y.; Nguyen, T.K.; Bouazzi, Y.; Kanzari, M. Design of output-graded narrow polychromatic filter by using photonic quasicrystals. Phys. B Condens. Matter 2020, 582, 411918. [CrossRef]

17. Nozaki, K.; Baba, T. Quasiperiodic photonic crystal microcavity lasers. Appl. Phys. Lett. 2004, 84, 4875-4877. [CrossRef]

18. Belhadj, W. Properties of omnidirectional gap and defect mode of one-dimensional graphene-dielectric periodic structures. Opt. Quantum Electron. 2020, 52, 1-20. [CrossRef]

19. Zeng, C.Z.C.; Luo, C.L.C.; Hao, L.H.L.; Xie, Y.X.Y. The research on magnetic tunable characteristics of photonic crystal defect localized modes with a defect layer of nanoparticle. Chin. Opt. Lett. 2014, 12, S11602-S311604. [CrossRef]

20. Kłos, J.W.; Krawczyk, M.; Dadoenkova, Y.S.; Dadoenkova, N.N.; Lyubchanskii, I.L. Photonic-magnonic crystals: Multifunctional periodic structures for magnonic and photonic applications. J. Appl. Phys. 2014, 115, 174311. [CrossRef]

21. Dadoenkova, Y.S.; Dadoenkova, N.N.; Lyubchanskii, I.L.; Kłos, J.W.; Krawczyk, M. Confined states in photonic-magnonic crystals with complex unit cell. J. Appl. Phys. 2016, 120, 073903. [CrossRef]

22. Dadoenkova, N.N.; Dadoenkova, Y.S.; Panyaev, I.S.; Sannikov, D.G.; Lyubchanskii, I.L. One-dimensional dielectric bi-periodic photonic structures based on ternary photonic crystals. J. Appl. Phys. 2018, 123, 043101. [CrossRef]

23. Panyaev, I.S.; Yafarova, L.R.; Sannikov, D.G.; Dadoenkova, N.N.; Dadoenkova, Y.S.; Lyubchanskii, I.L. One-dimensional multiperiodic photonic structures: A new route in photonics (four-component media). J. Appl. Phys. 2019, 126, 103102. [CrossRef]

24. Dionne, J.A.; Sweatlock, L.A.; Atwater, H.A.; Polman, A. Planar metal plasmon waveguides: Frequency-dependent dispersion, propagation, localization, and loss beyond the free electron model. Phys. Rev. B 2005, 72, 075405. [CrossRef]

25. Wu, J.-J.; Gao, J.-X. Temperature-dependent optical properties of defect mode in dielectric photonic crystal heterostructure containing a superconducting layer. Mater. Chem. Phys. 2016, 171, 91-96. [CrossRef]

26. Trabelsi, Y.; Ali, N.B.; Kanzari, M. Tunable Narrowband optical filters using superconductor / dielectric generalized Thue-Morse photonic crystals. Microelectron. Eng. 2019, 213, 41-46. [CrossRef]

27. Liu, Y.; Yi, L. Tunable terahertz multichannel filter based on one-dimensional superconductor-dielectric photonic crystals. J. Appl. Phys. 2014, 116, 223102. [CrossRef]

28. Aly, A.H. Metallic and Superconducting Photonic Crystal. J. Supercond. Nov. Magn. 2008, 21, 421-425. [CrossRef] 
29. Aly, A.H.; Elsayed, H.A.; Ameen, A.A.; Mohamed, S.H. Tunable properties of one-dimensional photonic crystals that incorporate a defect layer of a magnetized plasma. Int. J. Mod. Phys. B 2017, 31, 1750239. [CrossRef]

30. Arafa, A.H.; Hussien, E.S.; Sahar, E. Tunability of two dimensional n-doped semiconductor photonic crystals based on the Faraday effect. Opt. Express 2015, 23, 15038-15046.

31. Lin, Y.-T.; Chang, W.-Y.; Wu, C.-Y.; Zyryanov, V.Y.; Lee, W. Optical properties of one-dimensional photonic crystal with a twisted-nematic defect layer. Opt. Express 2010, 18, 26959-26964. [CrossRef]

32. Zyryanov, V.Y.; Myslivets, S.A.; Gunyakov, V.A.; Parshin, A.M.; Arkhipkin, V.G.; Shabanov, V.F.; Lee, W. Magnetic-field tunable defect modes in a photonic-crystal/liquid-crystal cell. Opt. Express 2010, 18, 1283-1288. [CrossRef]

33. D'Alessandro, A.; Asquini, R. Liquid Crystal Devices for Photonic Switching Applications: State of the Art and Future Developments. Mol. Cryst. Liq. Cryst. 2003, 398, 207-221. [CrossRef]

34. Trabelsi, Y.; Ben Ali, N.; Aly, A.H.; Kanzari, M. Tunable high Tc superconducting photonic band gap resonators based on hybrid quasi-periodic multilayered stacks. Phys. C Supercond. Its Appl. 2020, 576, 1353706. [CrossRef]

35. Yamamoto, A.; Takeshita, N.; Terakura, C.; Tokura, Y. High pressure effects revisited for the cuprate superconductor family with highest critical temperature. Nat. Commun. 2015, 6, 8990. [CrossRef]

36. Trabelsi, Y.; Kanzari, M. Omnidirectional reflection from deformed quasiperiodic one-dimensional photonic crystals in high frequency. Phys. Procedia 2009, 2, 947-951. [CrossRef]

37. Antonella D'Orazio, Infiltrated liquid crystal photonic bandgap devices for switching and tunable filtering. Fiber Integr. Opt. 2010, 22, 161-172.

38. Zyryanov, V.Y.; Gunyakov, V.A.; Myslivets, S.A.; Arkhipkin, V.G.; Shabanov, V.F. Electrooptical Switching in a One-Dimensional Photonic Crystal. Mol. Cryst. Liq. Cryst. 2010, 488, 118-126. [CrossRef]

39. Kowerdziej, R.; Parka, J.; Nyga, P.; Salski, B. Simulation of a tunable metamaterial with nematic liquid crystal layers. Liq. Cryst. 2011, 38, 377-379. [CrossRef]

40. Alihosseini, F.; Ahmadi, V.; Mir, A. Design and analysis of a tunable liquid crystal switch/filter with metallic nano-slits. Liq. Cryst. 2015, 32, 1638-1642. [CrossRef]

41. Lio, G.; Ferraro, A. LIDAR and Beam Steering Tailored by Neuromorphic Metasurfaces Dipped in a Tunable Surrounding Medium. Photonics 2021, 8, 65. [CrossRef]

42. Wu, C.-Y.; Zou, Y.-H.; Timofeev, I.; Lin, Y.-T.; Zyryanov, V.Y.; Hsu, J.-S.; Lee, W. Tunable bi-functional photonic device based on one-dimensional photonic crystal infiltrated with a bistable liquid-crystal layer. Opt. Express 2011, 19, 7349-7355. [CrossRef]

43. Bouazzi, Y.; Kanzari, M. Optical Fabry-Perot filter based on photonic band gap quasi-periodic one-dimensional multilayer according to the definite Rudin-Shapiro distribution. Opt. Commun. 2012, 285, 2774-2779. [CrossRef]

44. Trabelsi, Y.; Ali, N.B.; Elhawil, A.; Krishnamurthy, R.; Kanzari, M.; Amiri, I.S.; Yupapin, P. Design of structural gigahertz multichanneled filter by using generalized Fibonacci superconducting photonic quasicrystals. Results Phys. 2019, $13,10234$. [CrossRef]

45. Wang, Y.-Y.; Chen, L.-W. Tunable negative refraction photonic crystals achieved by liquid crystals. Opt. Express 2006, 14, 10580-10587. [CrossRef]

46. Vaveliuk, P.; Moraes, F.; Fumeron, S.; Matos, O.M.; Calvo, M.L. Structure of the dielectric tensor in nematic liquid crystals with topological charge. J. Opt. Soc. Am. A 2010, 27, 1466-1472. [CrossRef]

47. Da, H.-X.; Li, Z.Y. Manipulating Nematic Liquid Crystals-based Magnetophotonic Crystals. In New Developments in Liquid Crystals Book; Tkachenko, G.V., Ed.; I-Tech: Vienna, Austria, 2009; ISBN 978-953-307-015-5.

48. Asquini, R.; Fratalocchi, A.; D'Alessandro, A.; Assanto, G. Electro-optic routing in a nematic liquid-crystal waveguide. Appl. Opt. 2005, 44, 4136-4143. [CrossRef] 